\title{
Regrowth potential of shoot and of roots of Rhodes grass (Chloris gayana Kunth) after defoliation
}

\author{
A. Dovrat ${ }^{1}$, E. Dayan ${ }^{1}$ and H. van Keulen ${ }^{2}$ \\ 1Faculty of Agriculture, Hebrew University, Rehovot, Israel \\ 2 Center for Agrobiological Research, Wageningen, the Netherlands
}

Accepted: 4 June 1980

Key words: Rhodes grass, Chloris gayana Kunth, defoliation, shoot, roots

\section{Summary}

Field and pot trials are described in which the effect of different lengths of precutting periods and of cutting intervals on regrowth of shoot and of root were studied in cv. Katambora Rhodes grass.

The initial regrowth of the sward after a long (28 days) pre-cutting period was slower than after a short ( 7 days) pre-cutting period. The reduction of the residual leaf area index following the extension of the pre-cutting period, was associated with reduction in the number of tillers capable of regrowth after defoliation. Root weights decreased drastically following cutting when the initial root weight was high (long pre-cutting period), but little when the initial root weight was small (short pre-cutting period). The decrease in root weight lasted about 1 week after which it increased at more or less constant rate proportional to the increase of shoot weight irrespective of the length of the pre-cutting period. The lack of the capability of tillers to regrow after cutting was closely related with developmental stage of tillers. Since tillers of subtropical and tropical grasses have a tendency for early stem elongation, it was concluded that the relatively small number of sites available for regrowth in these grasses is the major deterrent for quick shoot growth of the sward after defoliation.

\section{Introduction}

In the framework of a joint Dutch-Israeli research project on 'actual and potential herbage production under arid conditions', crop growth under both rainfed and irrigated conditions was studied in a low rainfall region. One of the aims of the project was to develop, improve and test models for crop growth under the prevailing conditions (van Keulen, 1975; Dayan et al., 1981a).

Most published simulation models, such as BACROS (de Wit et al., 1978) tend 


\section{A. DOVRAT, E. DAYAN AND H. VAN KEULEN}

to treat the morphogenetic processes in the plant in an oversimplified way, since the main interest is the simulation of dry-matter accumulation during uninterrupted growth. When, however, exploitation takes place through cutting or grazing, as is the case with many grasses, morphogenetic parameters are of prime importance, since they have a decisive effect on the regrowth after defoliation. Such morphogenetic parameters require quantitative assessment when an effort is made to simulate dry-matter accumulation of a grass sward subjected to periodic defoliation (Dayan et al., 1981b).

In this study with Rhodes grass, special attention was paid to the fate of tillers, with respect to shoot and to root growth after defoliation, in swards composed of heterogeneous populations of tillers having different phenological state.

\section{Experimental}

\section{Field experiments}

A field of $3 / 4$ ha was seeded with Katambora Rhodes grass in the early spring of 1973 on a loamy sand soil at the Experimental Farm of the Faculty of Agriculture at Rehovot. During approximately 4 months of establishment, the field was mowed a number of times to obtain a more or less dense grass stand. In a randomized block design (plot size $7 \mathrm{~m} \times 24 \mathrm{~m}$ ), the following pre-cutting periods were imposed: 1, 2, 3 or 4 weeks, after which weekly harvests with Agria power mower (at $6-\mathrm{cm}$ cutting height) were made during 8 weeks from $9.8-\mathrm{m}^{2}$ subplots to determine dry-matter accumulation. There were 4 replications. Two representative samples of $0.5 \mathrm{~kg}$ each were taken from the cut foliage, weighed green and after drying (at $75^{\circ} \mathrm{C}$ in a forced-air oven during $24 \mathrm{~h}$ ) weighed again for determining the percentage of dry matter. The field was sprinkler-irrigated at night sufficiently for maintaining near field capacity $(0.05-0.1$ bar $)$ in the $0-20 \mathrm{~cm}$ soil layer. Moisture tension in the soil was determined using tensiometers. Fertilizer was applied as required to maintain in the $0-20 \mathrm{~cm}$ soil layer the following concentrations: $\mathrm{N}-50 \mathrm{mg} / \mathrm{kg}$ (with ammonium nitrate limestone), $\mathrm{P}-19 \mathrm{mg} / \mathrm{kg}$ (with single superphosphate) and $\mathrm{K}-\triangle \mathrm{F}$ equals $-12.6 \mathrm{~kJ} / \mathrm{mol}$ (with potassium chloride).

In another experiment, $1 / 3$ ha of a well-established 2 -year old field of Katambora Rhodes grass was subdivided into 4 blocks (replications) in early summer. In a randomized block design (plot size $14 \mathrm{~m} \times 14 \mathrm{~m}$ ), the following cutting intervals were imposed: $1,2,3$ or 4 weeks, during a period of 12 weeks. At weekly intervals, $6.7-\mathrm{m}^{2}$ subplots were harvested for determining dry-matter accumulation. Irrigation and fertilizer application was as in the formerly described experiment.

Tillers. All tillers above 6-cm cutting height from two $0.5 \mathrm{~m} \times 0.5 \mathrm{~m}$ areas per plot, chosen at random, were harvested weekly and separated by hand into the following categories: (1) vegetative, (2) with beginning of stem formation, (3) nonflowering elongated stem, (4) flowering stem, (5) flowering stem plus secondary vegetative tiller, and (6) flowering stem plus flowering secondary tiller. Their 
number, weight and leaf area were determined.

A regrowing tiller is defined as a tiller of which apex remains active after defoliation.

Amounts of roots. Soil samples, at intervals of $5 \mathrm{~cm}$ each down to $50 \mathrm{~cm}$, were taken with an auger $(6.5 \mathrm{~cm}$ diameter) at weekly intervals from 2 sites, chosen at random, in each plot and placed in aluminium tins. The soil samples containing the roots were placed in tapered glass vessels, through which slowly running water caused separation of most of the roots from the soil. Roots were collected, dried (at $75^{\circ} \mathrm{C}$ ) and weighed.

Distribution of roots. A hole measuring $2 \mathrm{~m} \times 2 \mathrm{~m} \times 1.5 \mathrm{~m}$ was dug in the field of the first experiment prior to seeding. A sturdy wooden frame lined with plywood walls, each provided with $0.4 \mathrm{~m}$ (wide) by $1.5 \mathrm{~m}$ (high) glass plate $(2 \mathrm{~mm}$ thick) as 'window', was carefully lowered into the hole so as to disturb soil texture as little as possible. A $10 \mathrm{~cm} \times 10 \mathrm{~cm}$ grid was marked on each window. Windows were always covered with a 2 -cm-thick layer of plastic foam for insulation. The hole was sheltered with a wooden cover. Once a week the window covers were removed and the number of apparent live roots, which had transversed the grid lines was counted.

\section{Pot experiments}

Pots filled with sand. Two young and rooted tillers were planted in white-painted plastic pots (10 litres), filled with a 2-mm layer of quartz sand, which was previously washed. Each pot was covered with 1.5 -cm-thick plastic foam with a hole in the centre $(4 \mathrm{~cm}$ diameter) through which the plants grew. This cover prevented evaporation from the sand surface and from runners to root. Pots were enfolded in 1.5-cm-thick plastic foam sheets for insulation and placed at $0.50 \mathrm{~m} \times 0.50 \mathrm{~m}$ distance between each other on benches in the open. Watering was done from a closed-circuit drip-irrigation system. Tubes fitted with one emitter per pot (which was mounted on the cover), obtained water by pumping it from at reservoir at 15min intervals during $15 \mathrm{~min}$. Surplus water drained into the reservoir by gravitation through outlets at the bottom of each pot. Irrigation water contained fullstrength Hoagland solution which was renewed weekly.

Plants, after establisment, were cut at 1-week intervals to a height of $11 \mathrm{~cm}$ above the cover surface during 4 weeks. Then, different lengths of pre-cutting periods were imposed, i.e., 1, 2, 3 or 4 weeks. The weight of shoot regrowth and of roots were determined by destructive harvesting at weekly intervals during 8 weeks. Roots were separated from shoot, washed over fine netting for the removal of sand, dried at $70^{\circ} \mathrm{C}$ during $24 \mathrm{~h}$, ground and weighed. There were 4 pots (replications) per treatment.

Pots filled with nutrient solution. Two young and rooted tillers were enfolded at their base with a 2 -cm-wide strip of plastic foam and firmly placed in a 2 -cm-wide 

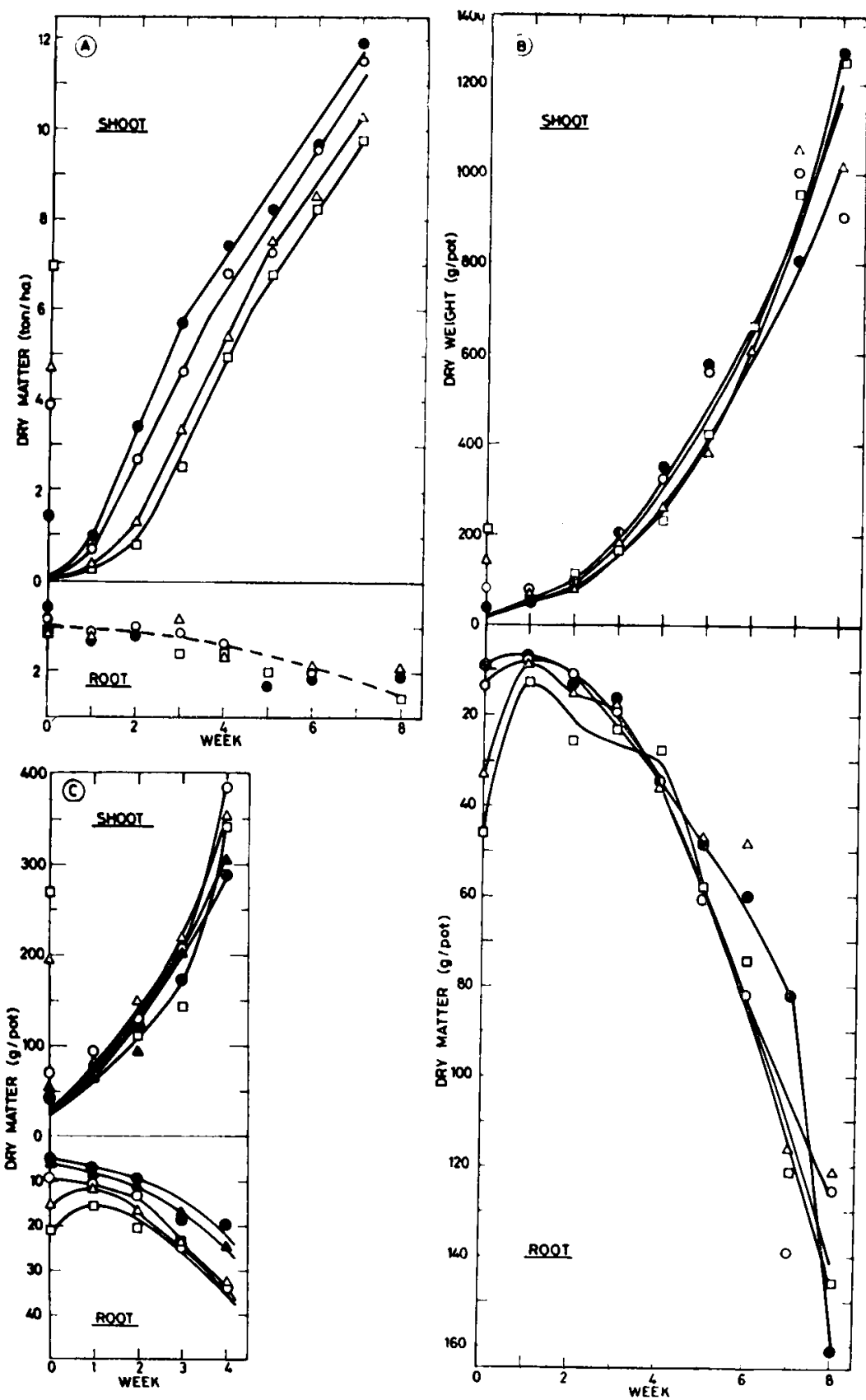

Fig. 1. Effect of length of different pre-cutting periods on the accumulation of shoot and of roots of Katambora Rhodes grass. $\mathrm{A}=$ field; $\mathrm{B}=$ pots filled with sand; $\mathrm{C}=$ pots filled with nutrient solution. Pre-cutting periods: - 1 week; $\Delta 1.5$ weeks; $\bigcirc 2$ weeks; $\triangle 3$ weeks; $\square 4$ weeks. 
hole of a 1.5 -cm-thick plastic cover. The covers were placed on 10-litre plastic pots filled with nutrient solution in which the roots immersed. The pots were painted white and enfolded in 1.5-cm-thick plastic foam sheets for insulation. Pots contained full-strength Hoagland solution, which was replaced once a week. Each day water was added to the pots and completed to a volume of 10 litres. Air from a compressor bubbled constantly through the nutrient solution. Treatments and determinations were identical to those mentioned above.

Leaf water potential. This was measured with the pressure bomb described by Scholander et al. (1965).

\section{Results}

\section{Shoot weight}

The crop growth rate of shoots in the field was exponential during the initial period of regrowth after cutting. The length of this period depended on the length of the pre-cutting period. After the initial period, the growth rate was linear and independent of the length of the pre-cutting period (Fig. 1A). After the long precutting period (28 days), it took about twice more time for the crop growth rate to reach maximum value than after the short pre-cutting period (7 days). This resulted in an approximately $20 \%$ higher total shoot weight after 8 weeks of growth, respectively. The growth rate of plants growing in pots was exponential throughout most of the regrowing period after cutting and was not affected by the length of the pre-cutting period (Fig. 1B and C).

The different course of regrowth of plants growing in the field compared with pots is due to the difference in above-ground space available for growth. Regrowth of plants in the field following cutting was exponential until the canopy was closed, after which growth became linear because of light penetration becoming limited. Regrowth of plants in pots remained exponential since open space between pots favoured light penetration and foliage could outgrow the boundaries of the pots.

\section{Leaf area index and composition of shoot}

After the long period of growth prior to cutting, leaf area index (LAI) slowly increased, whereas after the short period of growth, LAI almost immediately reached maximum rate, analogous to the course of crop growth rate. Since initial regrowth after the short pre-cutting period is mainly composed of expanding leaf blades, it was not surprising to find that leaf area and weight of shoot per unit area of ground were closely related (Fig. 2). The main cause of the low residual LAI following the extension of the length of the pre-cutting period was the reduction in the number of tillers capable of regrowth after cutting (Fig. 2). 


\section{A. DOVRAT, E. DAYAN AND H. VAN KEULEN}

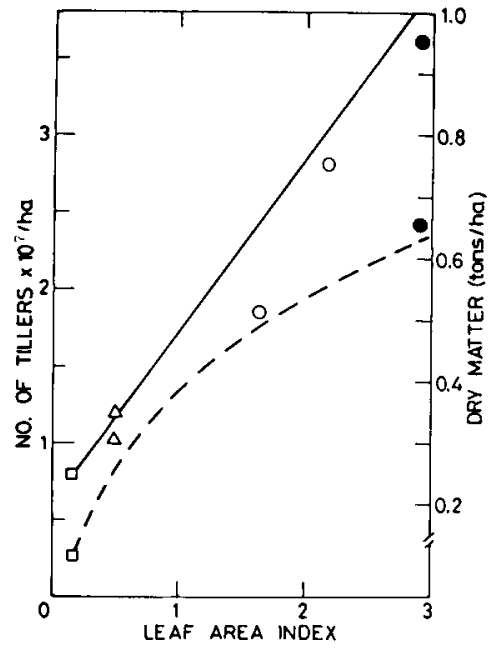

Fig. 2. Relationship between leaf area index (after 1 week of regrowth following cutting), the number of tillers and the weight of shoot of field-grown Katambora Rhodes grass. Pre-cutting periods: 1 week; $\bigcirc 2$ weeks; $\triangle 3$ weeks; $\square 4$ weeks.

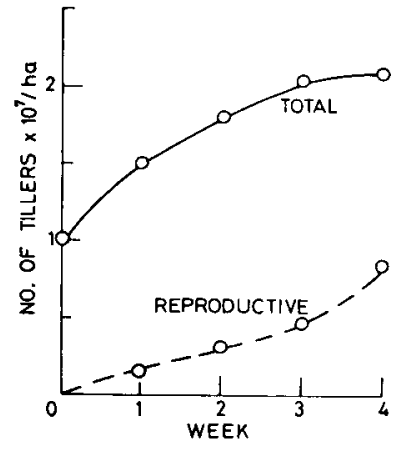

Fig. 3. Total and reproductive tillers during regrowth of field-grown Katambora Rhodes grass.

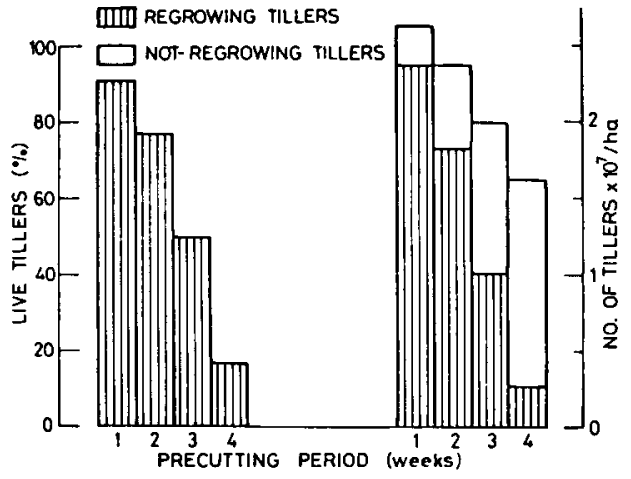

Fig. 4. Effect of different pre-cutting periods on the number and percentage of tillers capable of Rhodes grass. Counts were made 5 days after cutting. 
Developmental stage, number and weight of tillers

Tillers, after initiation, grow vegetatively (leaf blades only), after which they pass through various stages of reproductive growth, the start of which is stem formation and is completed by flowering and by the formation of secondary tillers. Generally, in the field, the total number of tillers per unit area increased after cutting, but soon tended to reach a maximum value, whereas the percentage of reproductive tillers steadily increased (Fig. 3 ).

A very marked effect of the length of the pre-cutting period on the percentage of tillers capable of regrowth was found. When cutting the sward of 1-week-old grass, approximately $90 \%$ of the tillers were capable to regrow, whereas in the 4-week-old sward only approximately $20 \%$ regrew (Fig. 4). The differential capability of tillers to regrow after cutting was found to be associated with their developmental stage at the time of cutting. When swards of different age were cut (i.e., relatively small number of reproductive tillers in the 1-week-old stand, but a relatively big number of reproductive tillers in the 4-week-old stand), a highly significant negative correlation was found between number of reproductive tillers prior to cutting and number of tillers capable of regrowth after cutting (Fig. 5).

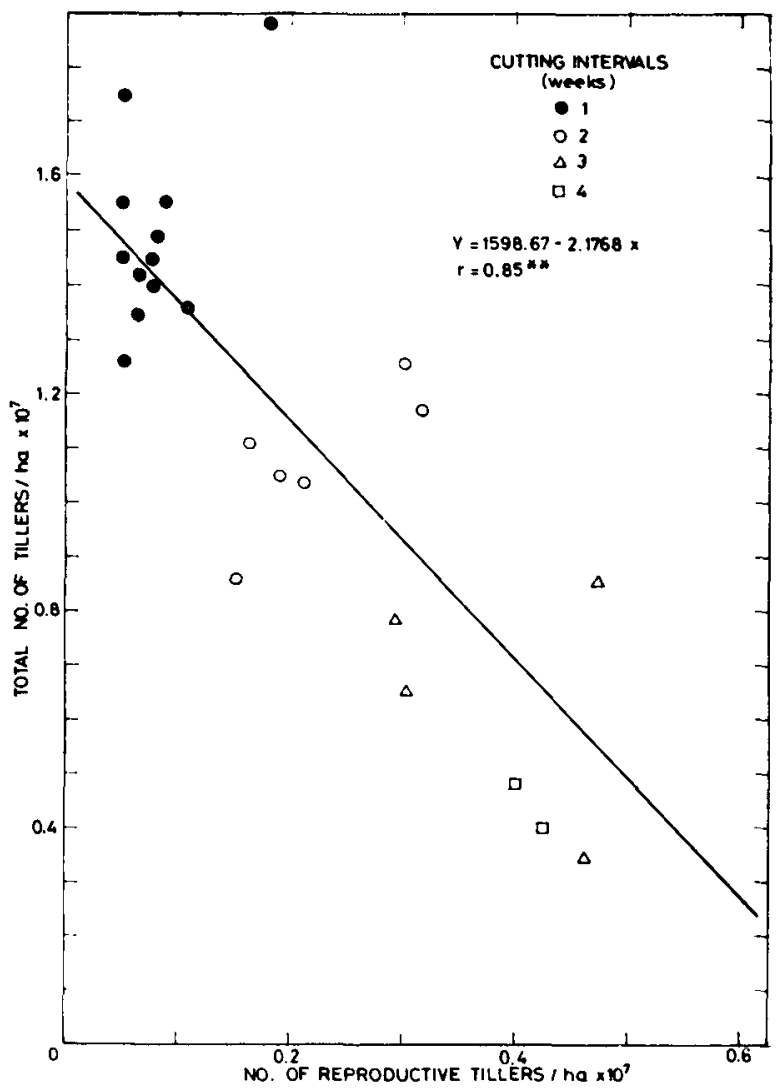

Fig. 5. Relationship between the number of reproductive tillers prior to cutting and the number of tillers capable of regrowth after cutting in Katambora Rhodes grass. Counts were made 1 week after cutting. 


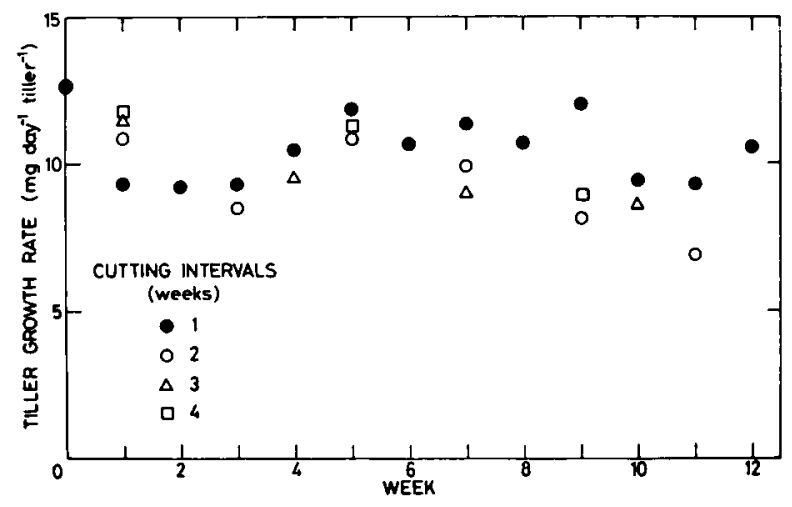

Fig. 6. Average daily growth rate of tillers during the 1 st week of regrowth after cutting.

The mean growth rate of tillers during the first week after cutting was approximately $10 \mathrm{mg}^{-1 a y}{ }^{-1}$ tiller-1 and was little affected by the length of the pre-cutting period (Fig. 6). Generally, somewhat higher, but not significantly, was the growth rate of tillers regrowing in swards cut at 1 -week intervals. This higher growth rate was apparently caused by the regrowth originating from the greater amount of leaf tissue which was left after cutting, compared with longer intervals. Regrowth after cutting swards older than 1 week was mostly from new tillers.

The association between number of tillers and crop growth rate lessened with time (Fig.7). Initial dry matter accumulation of regrowing shoot after cutting linearly increased with the number of tillers capable of regrowth. Thus, after 1 week of growth, the yield of swards with a high number of regrowing tillers was greater than with low number of regrowing tillers and the number of tillers was positively correlated with yield. This also was true for subsequent growth during

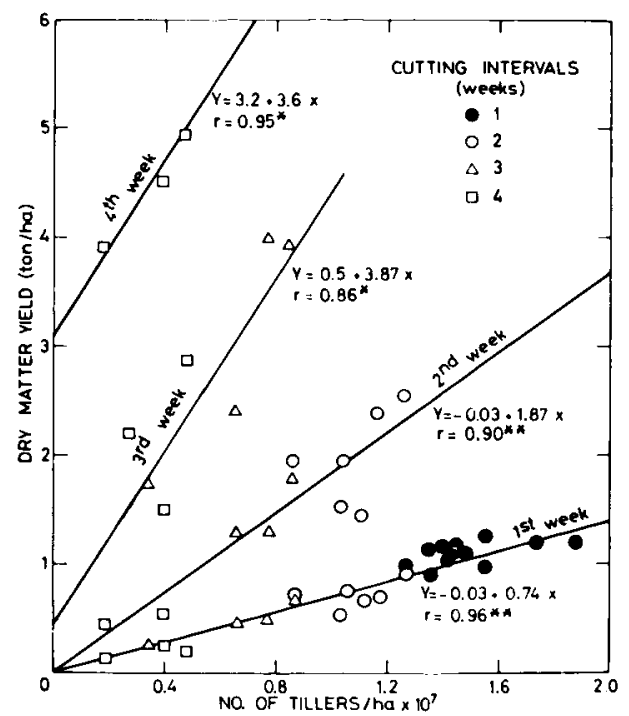

192
Fig. 7. Regression of number of regrowing tillers after cutting on the growth rate per week of Katambora Rhodes grass when cut at different intervals. 
the 2 nd, 3rd and 4th week. Since the growth of shoot gradually increased with time, the slope of the regression curves became steeper (until the 3rd week) after which during the 4th week no further increase in shoot growth rate occurred, because the leaf canopy became closed. Further it should be noted, that the intercepts of the regression curves became larger with time indicating that the growth rate of shoot was also affected by factors not related to the initial number of tillers capable of growth after cutting (i.e., new tillers), but present during the 1 st week of regrowth.

\section{Root weight}

The amount of roots, washed from soil core samples taken from the field, more than doubled during the 8 -week period of undisturbed growth following cutting (Fig. 1A). This increase in root weight was not affected by different lengths of the pre-cutting period. The lack of significant differences between the treatments is apparently the result of considerable variability and inaccuracies common to the method by which roots were sampled.

On the other hand, the observations from profiles of vertical root distribution revealed that different pre-cutting periods appreciably affected the number of live roots especially in the $0-20 \mathrm{~cm}$ deep soil layer (Fig. 8). The number of roots of plants in the sward which was cut once a week was more than twice lower than in swards cut less frequently. When the pre-cutting period was longer than 1 week, an immediate decrease in the number of live roots following cutting was observed whereas in the 1-week cutting frequency no decrease occurred (Fig. 8B, C and D). This decrease in the number of roots was the result of death and decomposition of roots. Approximately 14 days after the day of cutting, the original number of live roots was more or less restored after which it increased rapidly.

The weight of roots of plants growing in pots filled with sand increased proportionally with shoot weight (Fig. 1B). The removal of shoot by cutting, after different periods of growth, caused instant decrease of root weights. The largest decrease was in pots where the initial root weights were relatively high (4-week pre-cutting period) and the smallest to no decrease was in pots where the initial root weights were relatively small (1-week pre-cutting period). The decrease in root weight lasted for about 1 week after which it increased at a more or less constant rate without significant differences between treatments.

Similar results were obtained from plants growing in pots filled with nutrient solution, although the decrease in root weight after cutting was less pronounced and lasted longer. In plants defoliated after 1-2 weeks of growth no decrease in root weight was found (Fig. 1C).

\section{Leaf water potential}

The removal of shoot by cutting caused instant increase of the leaf water potential of the regrowing shoot compared with the intact shoot before cutting, which lasted from 8 to 12 days depending on the growth medium (i.e. field, pots filled 

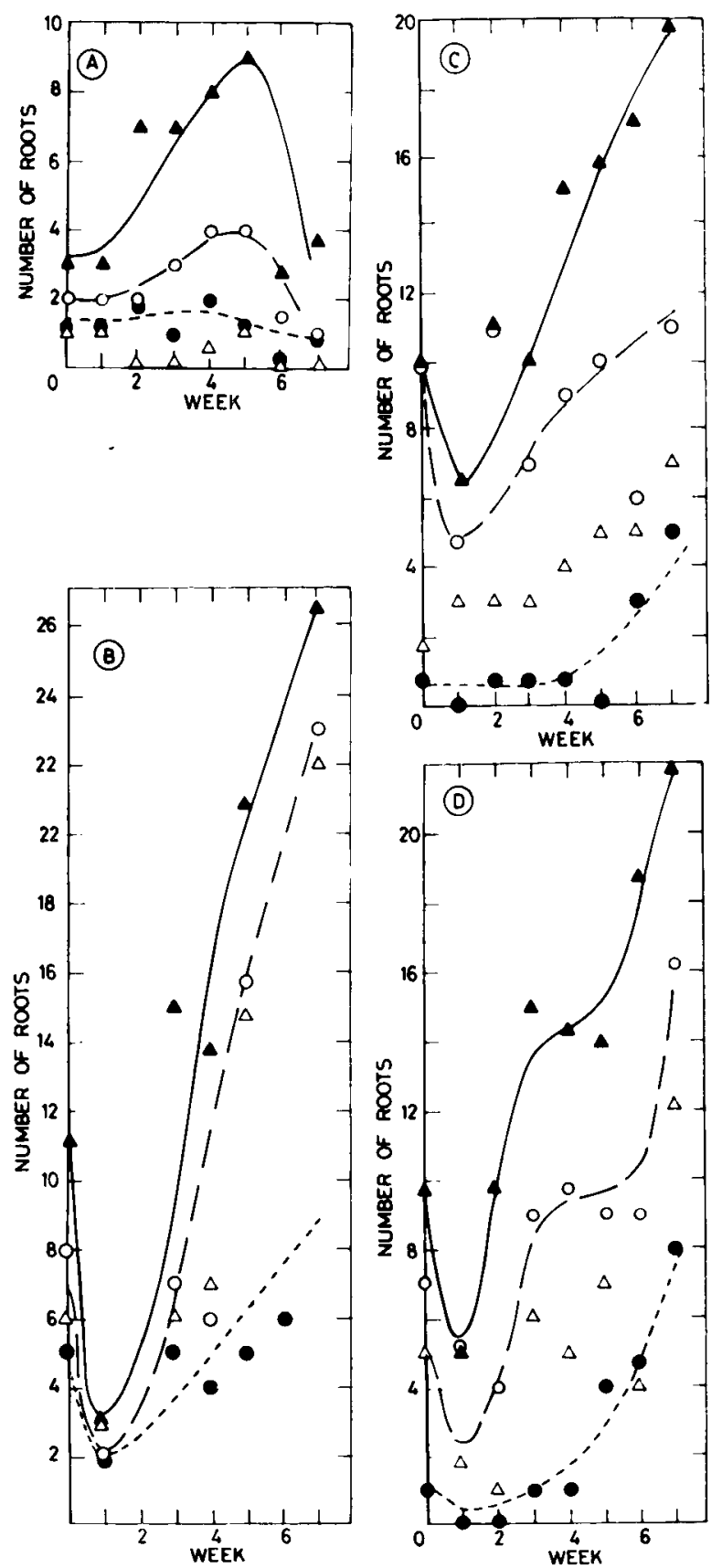

Fig. 8. Effects of different pre-cutting periods on the number of vertically-growing live roots at different soil depths of regrowing Katambora Rhodes grass in the field. Pre-cutting periods: $\mathrm{A}=1$ week; $\mathrm{B}=2$ weeks, $\mathrm{C}=3$ weeks; $\mathrm{D}=4$ weeks.

Soil depth: $\Delta \longrightarrow \Delta 10 \mathrm{~cm} ; \mathrm{O}-\mathrm{\Delta}-\mathrm{O} 20 \mathrm{~cm} ; \triangle 30 \mathrm{~cm} ;-\longrightarrow 50 \mathrm{~cm}$. 

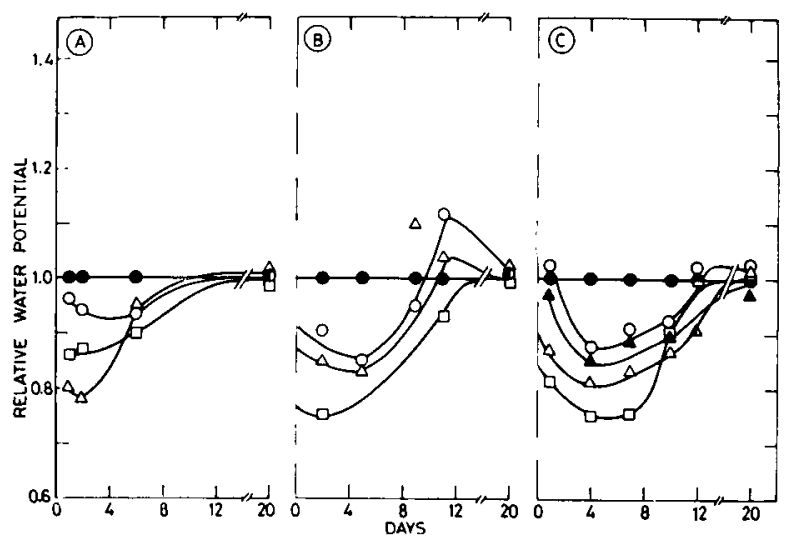

Fig. 9. Effect of different pre-cutting periods on the relative leaf water potential of regrowing shoot of Katambora grass. $\mathrm{A}=$ field; $\mathrm{B}=$ pots filled with sand; $\mathrm{C}=$ pots filled with nutrient solution. Pre-cutting periods: - 1 week; $\Delta 1.5$ week; $\bigcirc 2$ weeks; $\triangle 3$ weeks; 4 weeks.

with sand or with nutrient solution). The relative increase of leaf water potential in regrowing shoot was greater in plants which, prior to cutting, grew undisturbedly during 28 days compared with 7 days of growth (Fig. 9).

\section{Discussion}

It was found that prolonging the period of time prior to cutting decreased the rate of initial regrowth of the sward. Thus, more time was required for the sward to attain maximum growth than the short pre-cutting period. Factors which are known to affect the rate of growth of tillers after defoliation (i.e. mowing, cutting or grazing) are usually related to: (1) the availability of nutrients and/or reserve materials in the roots and stubble, (2) the altered shoot to root ratio and (3) the size of the residual leaf area and the number of sites available for growth per unit of ground area.

Previous work with Rhodes grass showed that the amounts of total non-structural carbohydrates in the shoot, stubble and roots are relatively low (Dovrat \& Cohen, 1970; Dovrat et al., 1972; Kipnis et al., 1977) when grown under conditions which favour rapid growth. It was also found that cutting frequency did not appreciably affect the level and the composition of total non-structural carbohydrates in either roots or stubble. So it is assumed that the availability of total non-structural carbohydrates in roots and stubble can hardly be the major cause for slow initial regrowth of the sward after the long interval compared with the short interval between cuttings.

Major differences in the shoot to root ratio, following different cutting regimes, neither appreciably affected the growth rate of tillers. If regrowth was affected by the sudden imbalance between shoot and roots following cutting, and the surplus of root relative to shoot would have functional meaning, than we would have 
expected more regrowth from tillers having a great amount of roots at their disposal (after the long cutting interval) compared with the small amount of roots (after the short cutting interval) (Brouwer, 1963). However, no appreciable difference in the initial rate of growth of individual tillers was found between the treatments. Neither the leaf water potential, which is considered to be a measure of the functional activity of roots (Brouwer \& de Wit, 1968), of regrowing plants was found markedly different between treatments, except slight differences during a short period after cutting only.

The increase of the leaf water potential of the regrowing tillers after cutting was much less than was expected from the alledged drastic change in the shoot to root ratio. However, in fact, measurements were made using young elongating tillers of which shoot to root ratio was relatively little affecting by cutting. Within a few days after cutting the leaf water potential decreased to values which were close to those before cutting, indicating that the original shoot to root ratio had been restored.

A plausible explanation for the lack of relationship between the alledged change in the shoot to root ratio and the growth rate of shoot is the differential death of roots after cutting. That is, the roots of tillers incapable of regrowth die, whereas the roots of tillers capable of growth remain alive and continue to grow. In other words, regrowing plants retained amounts of (live) roots proportional to the number of tillers capable of growth after cutting.

Temporary cessation of root growth following cutting is a common feature in grasses (Davidson \& Milthorpe, 1965, Ennik, 1966; Evans, 1972), including Rhodes grass (Crider, 1955). Our experiments confirmed these findings and additionally showed, that after cutting a mature sward (with a high percentage of reproductive tillers), death of roots was greater than after cutting a less mature sward (with a low percentage of reproductive tillers).

Death of roots soon follows after removal of the reproductive tiller. The rate of root decay was found dependent on the medium in which the roots grew. In the pots with sand, root decay was quicker than in the field and/or in the nutrient solution. This probably is caused by the more favourable conditions for decay (aeration, high temperatures) in the sand compared with the field or the nutrient solution.

Number of sites available for regrowth and the size of the residual leaf area index appeared to be major factors determining the rate of shoot growth, since neither the availability of reserve material in stubble and roots, nor the shoot to root ratio were found to have significant effects on regrowth. Positive correlation between the rate of shoot regrowth and the size of the residual leaf area index after cutting is also reported in timothy (Jewiss, 1972), ryegrass (Williams, 1970) and in other grasses.

Death of tillers after cutting seems mainly dependent on their phenological state. During growth of a sward, the weight of shoot increases because of both, the weight increase of individual tillers and the increase in the number of tillers. However, as much as the weight of tillers, associated with reproductive growth increased, the rate of newly formed tillers decreased. Inhibition of tillering (or 
suppression of auxillary bud development) during reproductive growth is wellknown in grasses (Kirby \& Faris, 1972; Jewiss, 1972). This is not only explained on the basis of apical dominance exerted by the reproductive tiller, but seems also related to light environment (Langer et al., 1964; Davies, 1969). Tillering in our field swards was markedly suppressed during reproductive growth, whereas in pots the formation of new tillers was much less affected.

Lack of capacity of a tiller to regrow after cutting (after which death follows) is mainly caused by the removal of the apical meristem with defoliation. Stem elongation can take place when the plane of cutting is above the apex; however, if the apex is removed with cutting, the stem of the tiller cannot further elongate. Inflorescence initiation and/or stem elongation seems to occur very early in the developing tiller of Rhodes grass, which makes young tillers particularly vulnerable to lack of regrowth after cutting (Kipnis et al., 1977). The precise morphological stage at which regrowth ceases after cutting was not determined by us, but all tillers which were identified as not having leaf blades only (reproductive tillers), failed to regrow. However, also part of tillers belonging to the "leaf blades only' category were observed to cease growth after cutting. These may have been tillers of which all leaves had reached full expansion (Sharman, 1947). Or, otherwise the drastic change in microclimate following the removal of shoot may have affected regrowth of young vegetative tillers (Williams, 1970). Additional causes of cessation of growth after cutting, such as physiological shock following drastic change in shoot to root ratio (Throughton, 1967; Evans, 1972) and accumulation of harmful metabolites (MacLeod, 1965; Teel, 1962), are also suggested.

Lack of regrowth and death of reproductive tillers after cutting occurred both in the field and in plants growing in pots, although young tillers in the latter retained a greater potential for regrowth. This may be related to the less extreme change in microclimate and radiation regime around the base of plants in pots compared with sward conditions. Furthermore, plants in plots showed a spreading growth habit, resulting in a more favourable light regime, whereas plants which grew in the sward had upright growth habit. Because of the greater regrowth potential of tillers of plants in pots, the difference in shoot growth rate after cutting between different pre-cutting periods was less pronounced than in field swards.

Since tillers of many subtropical and tropical grasses have a tendency of early stem elongation it is concluded that the relatively small number of sites available for regrowth after cutting is the major deterrent for quick shoot growth of the sward after defoliation. Also grasses with culmed vegetative tillers (Hyder, 1972) will be prone to slower regrowth after cutting than grasses with culmless vegetative tillers.

\section{Acknowledgments}

Prof. C. T. de Wit and Dr Th. Alberda's inspiration and interest in this study are gratefully acknowledged. The research was sponsored by the International Technical Aid of the Dutch and Israeli Ministries of Foreign Affairs. 


\section{A. DOVRAT, E. DAYAN AND H. VAN KEULEN}

\section{References}

Brouwer, R., 1963. Some aspects of the equilibrium between overground and underground plant parts. Jaarb. Inst. biol. scheik. Onderz. LandbGewassen: 31-39.

Brouwer, R. \& C. T. de Wit, 1968. A simulation model of plant growth with special attention to root growth and its conseuqences. In: W. J. Whittington (Ed.), Root Growth. Butterworth, London.

Crider, F. G., 1955. Root stoppage resulting from defoliation of grass. USDA tech. Bull. 1102.

Davidson, J. L. \& F. L. Milthorpe, 1965. Carbohydrate reserves in the regrowth of cocksfoot. J. Br. Grassld Soc. 20:15-18.

Davies, I., 1969. The influence of management on tiller development and herbage growth Welsh Pl. Breed. Str. tech. Bull. 3:1-121.

Dayan, E., H. van Keulen \& A. Dovrat, 1981a. Experimental evaluation of a crop growth simulation model. A case study with Rhodes grass. Agro-Ecosystems (submitted).

Dayan, E., H. van Keulen \& A. Dovrat, 1981b. Tiller dynamics and growth of Rhodes grass after defoliation: A model named TILDYN. Agro-Ecoystems (submitted).

Dovrat, A. \& Y. Cohen, 1970. Regrowth potential of Rhodes grass as affected by nitrogen and defoliation. Proc. 11th Int. Grassl. Congr. (Surfers Paradise) 552-554.

Dovrat, A., B. Deinum \& J. G. P. Dirven, 1972. The influence of defoliation and nitrogen of the regrowth of Rhodes grass. 2. Etiolated growth and nonstructural carbohydrate content. Neth. J. agr. Sci. 20:97-103.

Ennik, G. C., 1966. Influence of clipping and soil fumigation on shoot and root production of perennial ryegrass and white clover. Jaarb. Inst. biol. scheik. Onderz. LandbGewassen: 11-18.

Evans, P. S., 1972. Root growth of Lolium perenne. 3. Investigation of the mechanism of defoliation-induced suppression of elongation. N.Z. Jl. agric. Res. 15:347-355.

Hyder, D. N., 1972. Defoliation in relation to vegetative growth. In: V. B. Younger \& C. M. McKell (Ed.), The Biology and utilization of grasses. Academic Press, London.

Jewiss, O. R., 1972. Tillering in grasses - its significance and control. J. Br. Grassld Soc. 27:65-82.

Keulen, H. van, 1975. Simulation of water use and herbage growth in arid regions. Simulation Monograph. Pudoc, Wageningen.

Kipnis, T., A. Dovrat \& S. Lavee, 1977. Morphological and physiological aspects of regrowth of Rhodes grass after cutting. Agric. Res. Org. Bull. 173. Bet Dagan, Israel, pp. 127. (Hebrew with English summary).

Kirby, E. J., \& D. G. Faris, 1972. The effect of plant density on tiller growth and morphology in barley. J. agric. Sci., Camb. 78:281-288.

Langer, R. H. M., S. M. Ryle \& O. R. Jewiss, 1964. The changing plant and tiller populations of timothy and meadow fescue swards. J. appl. Ecol. 1:197-208.

MacLeod, L. B., 1965. Effect of nitrogen and potassium fertilization on the yield, regrowth and carbohydrate content of storage organs of alfalfa and grasses. Agron. J. 57:345-350.

Scholander, P. F., H. T. Hammel, E. D. Bradstreet \& E. A. Hemmingsen, 1965. Sap pressure in vasicular plants. Science 148:339-346.

Sharman, B. C., 1947. The biology and developmental morphology of the shoot apex in Gramineae. New Phyt. 46:20-34.

Teel, M. R., 1962. Nitrogen-potassium relationships and biochemical intermediates in grass herbage. Soil Sci. 93:50-55.

Throughton, A., 1967. The effect of mineral nutrition on the distribution of root growth in Lolium perenne. Ann. Bot. N.S. 31:447-454.

Williams, R. D., 1970. Tillerig in grasses cut for conservation with special reference to perennial ryegrass. Herb. Abstr. 40:383-388.

Wit, C. T. de et al., 1978. Simulation of assimilation, respiration and transpiration of crops. Simulation Monographs. Pudoc, Wageningen. 